\title{
Development of a Monitoring System for Smartphone Overuse
}

\author{
Jiang $\mathrm{Li}^{1,2,3,4^{*}}$, Ass Prof Dr med; Siguo Bi ${ }^{5^{*}}, \mathrm{PhD}$; Jiao-Er Ding ${ }^{1}$, MA, MPH; Yukun Lan ${ }^{1}, \mathrm{MA}, \mathrm{MPH}$; Hua Fu ${ }^{1}$ \\ ${ }^{1}$ School of Public Health, Fudan University, Shanghai, China \\ ${ }^{2}$ Health Communication Institute, Fudan University, Shanghai, China \\ ${ }^{3}$ The Key Laboratory of Public Health Safety, Fudan University, Shanghai, China \\ ${ }^{4}$ Fudan-Pudong Preventive Medicine Institute, Shanghai, China \\ ${ }^{5}$ School of Computer Science, Fudan University, Shanghai, China \\ *these authors contributed equally
}

\section{Corresponding Author:}

Jiang Li, Ass Prof Dr med

School of Public Health

Fudan University

PO Box 248

138 Yixueyuan Road

Shanghai,

China

Phone: 86154237509

Email: lijiang_fd@fudan.edu.cn

\section{Abstract}

Background: Smartphone overuse has become an epidemic public health concern around the world. Nowadays, the measurement of smartphone overuse, which comes to be known as smartphone addiction, is still relying on self-reported questionnaire. However, this leads to inaccuracy and it cannot perform continual measurement.

Objective: The aim of this study is to develop an IT based system monitoring daily usage behavior of smartphone automatically, which was named Smartphone Overuse Monitoring System (SOMS).

Methods: The monitoring system consists of an Android Smartphone application (SOMS App) and a web application server. The App was designed to fulfill the following core functions: 1. To collect users' general demographic data and identify the IMEI of smartphones; 2. To monitor using behavior and assess smartphone overuse; 3. To give instant feedback to users. The web server stores the data collected by the App and execute statistical analysis.

Results: We invited 11 participants to test the SOMS. The users were asked to fill out a short questionnaire at the first logon, which includes demographic information, such as name, sex, age etc. The SOMS App recorded the smartphone behavior as follows: power on/off, call in/out, screening on/off/unlock, programs usage. The data of program usage include which app and how long it was used. Once a day, the participants received a notification of the smartphone usage statistics for the last 24 hours. The users can also find key messages from the interface of the SOMS App, such as the top 10 most frequently and longest used Apps. Moreover, the SOMS App draws a fragment map, which illustrates the interrupted daily life by smartphone.

Conclusions: The monitoring system can tally the length and frequency of smartphone use and analyze the most influential Apps on people's daily life. It is not only significant for the screening of smartphone overuse but also for the development of intervention strategy.

(iproc 2017;3(1):e7) doi: 10.2196/iproc.8305

\section{KEYWORDS}

activity monitoring; app; behavior, addictive/*prevention \& control; psychometrics; smartphone

\section{Multimedia Appendix 1}

Full poster.

[PDF File (Adobe PDF File), 397KB-Multimedia Appendix 1] 
Edited by T Hale; this is a non-peer-reviewed article. Submitted 27.06.17; accepted 22.08.17; published 22.09.17.

Please cite as:

Li J, Bi S, Ding JE, Lan Y, Fu H

Development of a Monitoring System for Smartphone Overuse

iproc 2017;3(1):e7

URL: http://www.iproc.org/2017/1/e7/

doi: 10.2196/iproc.8305

PMID:

(CJiang Li, Siguo Bi, Jiao-Er Ding, Yukun Lan, Hua Fu. Originally published in Iproceedings (http://www.iproc.org), 22.09.2017. This is an open-access article distributed under the terms of the Creative Commons Attribution License (https://creativecommons.org/licenses/by/4.0/), which permits unrestricted use, distribution, and reproduction in any medium, provided the original work, first published in Iproceedings, is properly cited. The complete bibliographic information, a link to the original publication on http://www.iproc.org/, as well as this copyright and license information must be included. 